www.jmscr.igmpublication.org

Index Copernicus Value: 79.54

ISSN (e)-2347-176x ISSN (p) 2455-0450

crossref DOI: https://dx.doi.org/10.18535/jmscr/v7i6.71

Journal Of Medical Science And Clinical Research

IGM Publication

An Official Publication of IGM Publication

\title{
Evaluation of Prescribing Pattern of Corticosteroids in Dermatology OPD in Tertiary Care Hospital
}

\author{
Authors \\ Dr Laxmi Bhagunde ${ }^{1}$, Dr S.B Tamboli ${ }^{2}$, Dr Rajat Chhabra ${ }^{3}$ \\ ${ }^{1}$ Tutor, Department of Pharmacology, HBT and R.N. Cooper Hospital and medical college, Mumbai, \\ Maharashtra \\ ${ }^{2}$ Professor and Head, Department of Pharmacology, Dr. Shankarrao Chavan Government Medical College, \\ Nanded, Maharashtra \\ ${ }^{3}$ Drug Safety Physician, Parexel, Chandigarh
}

\begin{abstract}
Background: Dermatological problems manifest as primary and secondary cutaneous complaints, which are particularly more common in India. The skin problems that are commonly found are acne, burn scars, dermatitis, psoriasis, scabies, vitiligo, pediculosis, herpes simplex infection, varicella, herpes zoster, erythema, urticaria, and so on. Skin diseases amenable to the effects of topical corticosteroids are usually characterized by inflammation, hyperproliferation, and/or immunologic phenomenon. Topical corticosteroids may also be effective in the treatment of skin symptoms, such as burning and pruritus. Successful treatment depends on an accurate diagnosis and consideration of the steroid's delivery vehicle, potency, frequency of application, duration of treatment, and side effects. Prescribing of drugs is an important skill, which needs to be continuously assessed and refined suitably and it reflects the physician's skill in diagnosis and attitude towards selecting the most appropriate cost-effective treatment. Contemplating the financial affliction of the treatment on the patients and government, prevailing disease prevalence, it is imperative to study the prescribing pattern of corticosteroids in dermatological OPD.

Objectives: To assess the prescribing pattern of corticosteroids and evaluate the monotherapy and utilization of coadministered drugs along with corticosteroids in skin conditions.

Material \& Methods: The study design was prospective, single centred, cross sectional observational study. Out of 1410 subjects studied, a sample size of 311.60 was calculated. 312 properly selected subjects with diagnosis made by the treating dermatologist for Skin related ailments requiring Corticosteroids prescription were recruited as study subjects. An initial preparatory phase was followed by data collection phase in Case record forms. The collected data was numerically coded and entered in Microsoft Excel 2007 and analysed and prescribing indicators were calculated by maintaining anonymity and privacy of respondents.

Results: In our findings, in a sample size of 312, it was seen that a total of 365 glucocorticoids were prescribed. The prescriptions showed generic name in 193 (52.9\%) cases as compared to 172 (47.1\%) prescriptions were done with tradel brand names. Foremost route used was topical application (76\%), whereas $24 \%$ of all corticosteroids prescribed were by oral/ parental route. The average number of drugs prescribed per prescriptions were 3.68, whilst the average corticosteroid prescribed was 1.17.

Conclusion: Prescribing by generic name should be encouraged. Prescribers should be encouraged for undertaking correct diagnosis, providing treatment following STGs (Standard Treatment Guidelines) and protocols. This will aid in improving the prescribing of corticosteroid drugs to a large extent.

Keywords: Corticosteroids, prescribing indicators, dermatological diseases.
\end{abstract}




\section{Introduction}

Topical corticosteroids have been used for over 60 years and their introduction was a milestone in dermatology, most commonly preferred are topical treatments for inflammatory dermatoses such as psoriasis and atopic dermatitis. Over the years, investigations have given fixated on approaches to enhance potency of the antiinflammatory and immunosuppressive dimensions of these drugs, whereas curtailing adverse effects. $^{(1)}$ Glucocorticoids are administered locally, via topical and intralesional routes and systemically, through intramuscular, intravenous, and oral routes. ${ }^{(2)}$ Topical corticosteroids are available in a diverse form - creams, ointments, lotions, gels, and, more currently, foam. The vehicle used significantly affects the agent's clinical action, potency, and suitability to the patient. Moreover, some vehicles are more appropriate for specific body areas. Determination of the apposite product relies on lesion area, doctor experience, price, and patient preference, particularly regarding vehicle. ${ }^{(3)}$ They are used to treat inflammatory skin conditions by suppressing the inflammatory reaction and relieving symptoms. Topical applications are preferable as the drug is delivered directly to the target organ. The dose can effortlessly be controlled according to response. ${ }^{(4)}$ Glucocorticoids (topical/systemic) are used solo or in combination with other drugs frequently. ${ }^{(5)}$

In 1985, WHO defined rational use of drug as, 'Patients receive medications appropriate to their clinical needs, in doses that meet their own individual requirements, for an adequate period of time, and at the lowest cost to them and their community'. ${ }^{(6)}$ Such studies form the essential exploratory tools to determine the role of drugs in the society. They give rise to a strong sociomedical and health economics, which form the fundamental in making health-care decisions. The information pertaining to drug usage patterns of corticosteroids is lacking especially in India. Keeping these facts in consideration, this study was undertaken in patients who were prescribed corticosteroids under the dermatology OPD of a tertiary care hospital to generate baseline data and analyse various aspects of drug prescribing practices.

\section{Materials and Methods}

The present study was undertaken with an objective to determine the prescription pattern of glucocorticoids in the treatment of inflammatory skin diseases in dermatology outpatient dermatology (OPD) of tertiary care hospital.

Study Design: Prospective, single centred, cross sectional observational study. STROBE (Strengthening the Reporting of Observational Studies in Epidemiology) guidelines were used. ${ }^{(7)}$

Study Period: January 2016 to July 2017.

Study Site: Dermatology OPD in a tertiary care hospital.

Study Population: All the patients with inflammatory skin disorders attending the dermatology outpatient department who were prescribed glucocorticoids.

\section{Sample Size \& Sampling Procedure}

The probability is known, the sample's statistics can be generalized to the population at large (at least within a given level of precision). ${ }^{(8)}$ Yamane provides a simplified formula to calculate sample sizes. A 95\% confidence level and $\mathrm{P}=0.5$ are assumed for the equation. Where $\mathrm{n}$ is the sample size, $\mathrm{N}$ is the population size, and e is the level of precision.

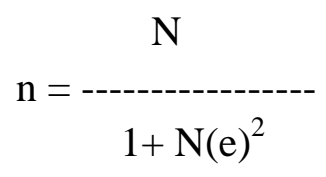

Thereby by applying the above formula, where our population of patient receiving corticosteroids via prescription during the study duration; $\mathrm{N}=$ $1410,95 \%$ confidence level and $\pm 5 \%$ precision level was applied. We get sample size of 311.60, so details of 312 patients were included in the study. ${ }^{(9)}$ 


\section{Inclusion Criteria}

- After obtaining approval and clearance from the institution ethical committee, all new patients aged 18-65 years enrolling in dermatology OPD for skin disease who were prescribed glucocorticoids for treatment.

\section{Exclusion Criteria}

- Patients unwilling for giving their consent, patients aged $<18$ or $>65$ years, patients already undergoing some other corticosteroid treatment for other than dermatological reasons, patients with underlying co-morbid conditions and pregnant and lactating women were excluded.

Ethical Considerations: Approval was obtained prior to the start of the study from Institutional Ethics Committee of Medical College, approved $\&$ registered with DCGI (Drug Controller General of India). All the data collected as a part of this study was kept strictly confidential and used for this study only.

\section{Study design- It consisted of following four phases}

\section{A) Preparatory phase-}

○ Seeking administrative approvalNovember to December 2015

- Constructing tools for data collection-

- Written inform consent form for subjects.

- CRF (Case Record Forms)

- Diagnosis by treating dermatologist.

- Drugs prescribed with information regarding name (brand/generic), dose, frequency, FDCs or not etc.

B) Phase of data collection-January 2016 to July 2017, in dermatology (Skin and Venereal Diseases) OPD.

- Detailed history was collected and entered in Case Record Form.

- Diagnosis made by the dermatologist and dermatologic evaluation and treatment regimen was under the discretion of treating dermatologist.

- The details about glucocorticoid drugs including brand/generic name, route, strength, dose, \& frequency of administration were then noted.

C) Phase of analysis- Data was numerically coded and entered in Microsoft Excel 2007 and analysed by SPSS (Statistical Package for Social Sciences) version 16 statistical software (IBM, Chicago, USA) by maintaining anonymity and privacy of respondents.

\section{D) Documentation phase-}

The analysed data was presented in the form of text, tables and graphs.

1. Information from the prescription: Demographic parameters, treatment details, diagnosis by the dermatologist.

2. Calculation of Core Indicators-

\section{Prescribing Indicators}

A Total Number of different drugs prescribed

* Average $=$

Total number of prescriptions surveyed.

\section{Number of GC's prescribed by generic name x 100 - Percentage $=$ \\ Total Number of drugs prescribed}

Statistical analysis: Descriptive and inferential statistical analysis has been carried out in the present study. Results on continuous measurements are presented on mean \pm SD (min$\max$ ) and results on categorical measurements are presented in number. (\%)

Reference citation: Reference citation was done by Vancouver referencing style.

\section{Results}

Out of 312 subjects taken up for the study, 168 $(53.85 \%)$ were males whereas $144(46.15 \%)$ were female. Figure no. 1 shows that amongst 312 
patients (males and females combined) most of the patients, 79 (25.3\%) were in age group of 2635 years, followed by 71 patients $(22.8 \%)$ in the age group of 36-45 years. The youngest subject was 18 years old and the oldest was 65 years old. The mean age in years \pm standard deviation of the study group individuals was $39.2 \pm 13.47$ (Mean \pm SD).

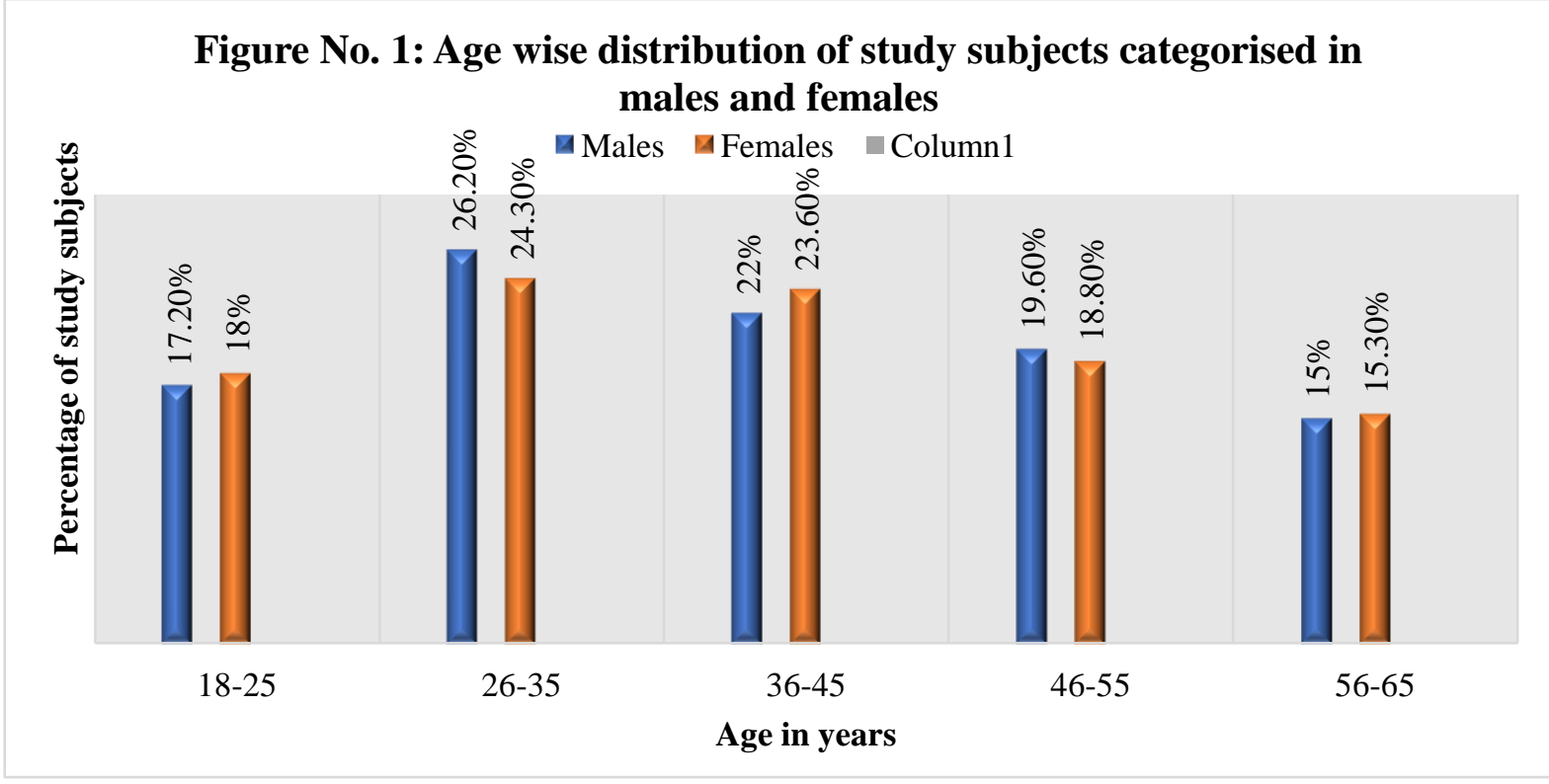

In our study we also observed that maximum number of the study subjects i.e. 178 (57\%) were residing in rural area, whereas $134(43 \%)$ were residing in urban area.

The study showed 34 out of $312(10.9 \%)$ study subjects were illiterate and $278(89.1 \%)$ of the study individuals were literate. We considered those subjects to be illiterate who couldn't read or write i.e. those subjects that have never learned to read or write.

In context to patients with family history, 142 (45.6\%) patients had relatives (family members) who were suffering from diabetes mellitus, whilst $158(50.6 \%)$ and $6(1.90 \%)$ patients gave a positive family history for hypertension and epilepsy/seizures respectively.
Figure no. 2 shows the disease pattern of study subjects; Eczema 94 (30.1\%) followed by Dermatitis $88(28.2 \%)$ represented majority of the skin ailments that were included in the study. Urticaria 15 (4.8\%), Plantar Keratitis 13 (4.2\%), PLE with Acne grade I/II 12 (3.85\%), Psoriasis 12 (3.85\%)- guttate, scalp and erythrodermic, Xerosis 10 (3.2\%), Acne keloidalis nuchae and Hypertrophic scar 15 (4.8\%), Unstable Vitiligo 8 (2.6\%), Senile Pruritis 8 (2.6\%), Acute Cutaneous Lupus Erythematosus 7 (2.2\%), Icthyosis Vulgaris 7 (2.2\%), Pemphigus Foliaceus 6 (1.9\%), Prurigo Nodularis 6 (1.9\%), Insect bite reaction 5 (1.6\%) and lastly Morphea and Hypertrophic Lichen Planus $3(1 \%)$ were the other conditions that were observed in our study. 


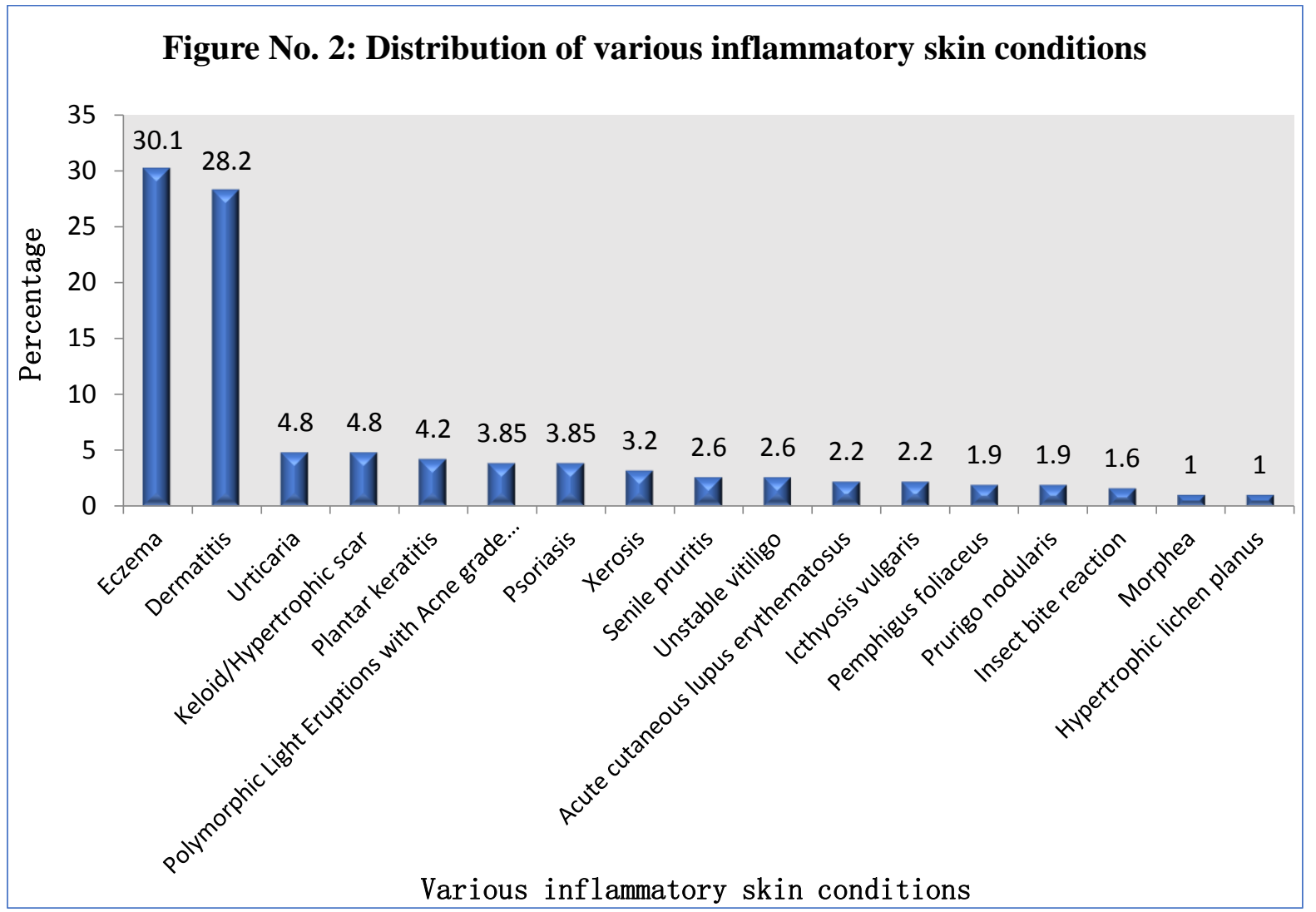

In the study we conducted of the prescription pattern of glucocorticoids in inflammatory skin disorders, with a sample size of 312 ; it was seen that a total of 365 glucocorticoids were prescribed. The prescriptions showed generic name in 193 (52.9\%) cases as compared to 172 (47.1\%) prescriptions were done with trade/ brand names.

Table no. 1 shows the most commonly prescribed drug by generic name is Betamethasone Cream (20 gm), contributing to $40.5 \%$ (148 out of 365 ) to the total glucocorticoids prescription. Foremost route used for drugs prescribed by generic name was by the topical application comprising 165 (85.5\%) of 193 glucocorticoid drugs prescribed by generic name whereas $28(14.5 \%)$ drugs were prescribed by other routes i.e., parental and oral.

Topically prescribed drugs were Betamethasone cream valerate $1 \% 20 \mathrm{gm}$ (148) and Mometasone Cream (17). Parentally prescribed were Inj. Hydrocortisone $100 \mathrm{mg}$ (12) and Inj. Dexamethasone. Least prescribed route was oral Tab. Prednisolone (10) and Tab. Beclomethasone (3).Of 193 drugs prescribed by generic names, $154(79.8 \%)$ were high potency corticosteroids.
Table No. 1: Prescription pattern of corticosteroid preparations (Generic name)

\begin{tabular}{|l|c|c|c|}
\hline Drug name & $\begin{array}{c}\text { No. of } \\
\text { patients }\end{array}$ & Route & Potency \\
\hline Betamethasone cream & 148 & Topical & High \\
\hline Mometasone cream & 17 & Topical & Medium \\
\hline Inj. Hydrocortisone & 12 & Parental & Low \\
\hline Inj. Dexamethasone & 3 & Parental & High \\
\hline Tab. Prednisolone & 10 & Oral & Medium \\
\hline Tab. Beclomethasone & 3 & Oral & High \\
\hline Total & 193 & & \\
\hline
\end{tabular}

Figure no. 3 shows that topically Cutisoft $1 \%$ cream (21) and Relysal 6\% ointment (21) were most common drugs prescribed by their trade names, whereas orally Tab. Omnacortil (30) and parentally Inj. Kenacort (24) were the most commonly prescribed drugs by oral and parental routes. Major route of all the drugs prescribed with trade name was of topical application i.e., $106(61.6 \%)$, whereas other routes comprised of $66(38.4 \%)$ prescriptions. Other corticosteroid drugs prescribed by their trade/ brand names were Topisal 6\% ointment (15), Sertacide B cream (9), Clop-G cream (12), Eumosone cream (4), SorvateC (3), Fucibet B cream (9), Onabet B cream (9), Tenovate ointment (3), and Betnesol Forte (12). 
Majority of the drugs prescribed by trade names and in combinations (Fixed Dose Combinations), were in the category of Ultra/ Very high 88 $(51.16 \%)$ followed by medium potency 54 $(31.40 \%)$ and least by Low/ mild potency 30 $(17.44 \%)$. By combining both generic and trade named prescriptions, we conclude that 242 (66.3\%) were high potency, $81(22.2 \%)$ were medium and $42(11.5 \%)$ were low/ mild potency drugs.

Table no. 2 shows the routes of administration, with $76 \%$ of corticosteroids being prescribed topically, whereas $24 \%$ of all corticosteroids prescribed by oral/ parental and intralesional route. The topical corticosteroids were in prescribed in 2 vehicle forms creams (229) and ointments (27).

Table no. 2: Routes of administration ofcorticosteroids

\begin{tabular}{|l|c|c|}
\hline Route & Number & Percentage \\
\hline Topical & 277 & $76 \%$ \\
\hline Oral/Parental & 88 & $24 \%$ \\
\hline Total & 365 & $100 \%$ \\
\hline
\end{tabular}

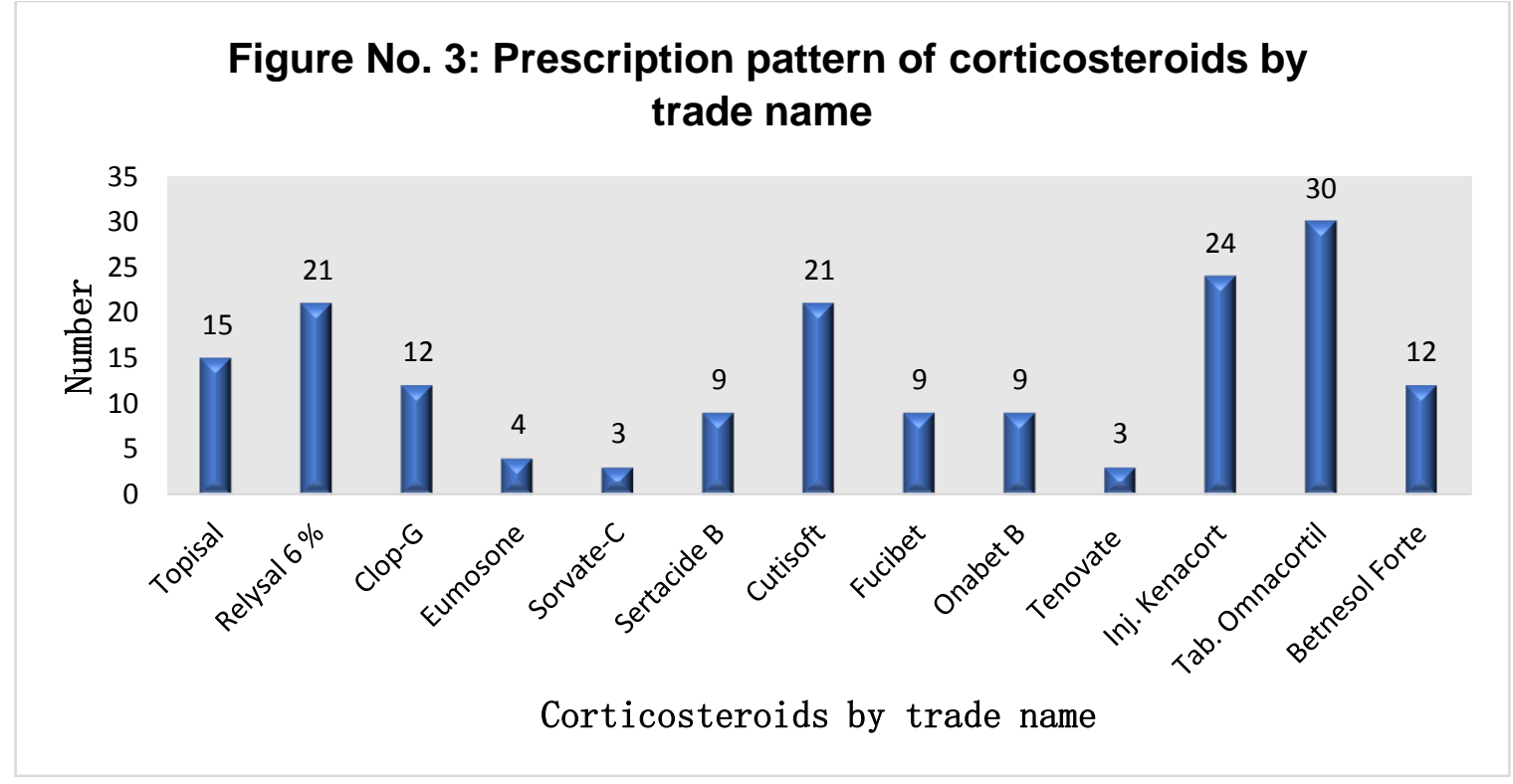

Table no. 3 shows that for total of 312 patients, a total of 785 drugs were co-administered. Antihistaminic drugs were the most common coadministered drug i.e., 241 (30.7\%), followed by antibiotics $151(19.23 \%)$. Other list of drugs that were co-prescribed were antacids (69), antiinflammatory (15), Multi-Vitamin B complex (43), Iron tablets (6), Calcium tablets (9). There were other drugs too that were prescribed which we listed under the category of Miscellaneous topical (209) and Miscellaneous oral (42). Tab. Cetrizine $10 \mathrm{mg} \quad 121 \quad(50.2 \%)$ and Tab. Chlorpheniramine maleate $4 \mathrm{mg} 66(27.4 \%)$ were the most common prescribed antihistaminics.

A total 151 prescriptions showed occurrence of various antibiotics. Major route for drugs prescription was by the oral route i.e., 99
$(65.56 \%)$ with 88 by generic name and 11 by their trade names, whereas $52 \quad(34.44 \%)$ were prescribed topically with 42 prescriptions holding generic name and 10 with trade name. in totality there was more multitude for generic name with $86.1 \%$ while $13.9 \%$ drugs were prescribed in their trade/ brand names. The drugs prescribed were as follows Tab. Azithromycin (42), Tab. Amoxycillin- Clavulinic acid (10), Tab. Terbinafine (18), Tab. Chloroquine (3), Tab. Doxycycline (6), Tab. Mebendazole (6), Tab. Flucanozole (3); were all prescribed by their generic names; Tab. Azithromycin (macrolide group of drugs) most commonly prescribed. Tab. Ocuvir (3), Tab. Taxim (5), Tab. Iverfast (3) were orally prescribed branded antibiotics, whereas Miconazole 2\% (29), Permethrin Cream (9) and 
Betadine mouthwash (4) were topical preparations with generic names.

Table No. 3: Drugs other than Glucocorticoids

\begin{tabular}{|l|c|c|}
\hline Drugs Name & $\begin{array}{c}\text { Number of } \\
\text { Patients }\end{array}$ & Route \\
\hline Antihistaminic & 241 & Oral/ Parental \\
\hline Antibiotics & 151 & $\begin{array}{c}\text { Oral/ Parental/ } \\
\text { Topical }\end{array}$ \\
\hline Antacids & 69 & Oral \\
\hline Anti-inflammatory & 15 & Oral \\
\hline $\begin{array}{l}\text { Multi Vitamin B- } \\
\text { complex }\end{array}$ & 43 & Oral \\
\hline Iron & 06 & Oral \\
\hline Calcium & 09 & Oral \\
\hline Miscellaneous Oral & 42 & Oral \\
\hline $\begin{array}{l}\text { Miscellaneous } \\
\text { Topical }\end{array}$ & 209 & Topical \\
\hline Total & 785 & \\
\hline
\end{tabular}

Finally, in Table no. 4 shows the contents of the prescription that were collected from the study subjects during the course of our study. The patient's details, with their name, age, sex, address and date of prescription was present in $100 \%$ of the study subjects. The occupation of the study subjects was seen on only $35.9 \%$ of prescriptions. The details pertaining to medication related information, dosage forms and dosage units were present on $100 \%$ of the prescriptions. Other details like the strength $(80.77 \%)$, frequency of administration (89.10\%), quantity (46,55), Duration $(95.5 \%)$ were also of present. The details of patients and prescriber were aptly present on most of the prescriptions owing to computerised system of OPD and inpatient patient registration and drugs dispensing.

From the all prescriptions which were evaluated it was found that average number of drugs prescribed per prescriptions was $\mathbf{3 . 6 8}$ and average number of corticosteroid drugs prescribed per prescriptions were 1.17.

Table no 4: Contents of the prescriptions $(n=312)$ Contents of the prescriptions Number Percentage Patient information:
a) Name of the patient
312
100
b) Age of the patient
312
100
c) Sex of the patient
312
100
d) Address of the patient
312
100
e) Occupation of the patient
112
f) Date of prescription
312

(\%)
Medication related information

\begin{tabular}{|c|c|c|}
\hline a) Dosage form of the drug & 312 & 100 \\
\hline b) Strength of the drug & 252 & 80.77 \\
\hline c) Dosage units of the drug & 312 & 100 \\
\hline d) Frequency of administration & 278 & 89.10 \\
\hline e) Quantity of drug & 144 & 46.15 \\
\hline f) Duration of treatment & 298 & 95.5 \\
\hline g) Instructions to the patients & 267 & 85.6 \\
\hline h) Hand writing legible & 243 & 77.88 \\
\hline \multicolumn{3}{|l|}{ Prescriber information: } \\
\hline a) Prescribing doctor: Specialist & Yes & \\
\hline \multirow{5}{*}{$\begin{array}{l}\text { b) Prescribing doctor: non- } \\
\text { specialist } \\
\text { c) Prescribing doctor name } \\
\text { mentioned } \\
\text { d) Prescribing doctor's signature } \\
\text { /initials }\end{array}$} & NA & \\
\hline & Yes & \\
\hline & NA & \\
\hline & Yes & \\
\hline & & \\
\hline $\begin{array}{l}\text { e) Registration number } \\
\text { mentioned }\end{array}$ & & \\
\hline
\end{tabular}

\section{Discussion}

The topical corticosteroids were introduced in early 1950s; since then, they are extensively prescribed medication in dermatology clinics. This requires essential care in the assortment of corticosteroid drugs for use and their dosage regimen. (10)

So, this study focuses to evaluate and analyse the prescription according to WHO core drug prescribing indicators. This was a cross sectional observational study carried out to evaluate the utilization of glucocorticoid drugs in tertiary care hospital.

The most common inflammatory conditions that were diagnosed, out of 312 study subjects, it was found that most of the diagnosed patients prescribed corticosteroids were having eczema 94 $(31.7 \%)$ and dermatitis $88(28.2 \%)$ and least integer of cases diagnosed were of morphea and hypertrophic lichen planus 3 (1\%). (Figure no.2) In a similar study done on 350 patients (prescriptions of patients attending dermatology OPD) by Sathendra Kasyap JV et al., most common encountered disease was eczema 175 $(50 \%)$ followed by psoriasis $36(10.3 \%)$ and Vitiligo $34(9.7 \%)$, other studies too were in concurrence with our study. ${ }^{(11,12)}$

Table no. 1 shows the prescription patterns of corticosteroids in a sample size of 312 . It was seen that a total of 365 glucocorticoids were prescribed. 
The prescriptions showed generic name in 193 (52.9\%) cases as compared to 172 (47.1\%) prescriptions were done with trade/ brand names. The table illustrates that most commonly prescribed drug by generic name is Betamethasone Cream (20 gm), contributing to $40.5 \%$ (148 out of 365 ) to the total glucocorticoids prescription. Topically prescribed drugs were Betamethasone cream valerate $1 \% 20$ gm (148) and Mometasone Cream (17). Parentally prescribed were Inj. Hydrocortisone $100 \mathrm{mg}$ (12) and Inj. Dexamethasone. Least prescribed route was oral Tab. Prednisolone (10) and Tab. Beclomethasone (3). Our findings correlate well with another study done by Mahendra Kumar Jaiswal et al. ${ }^{(13)}$

The table showing corticosteroid drugs prescribed by generic names, also reveal the significant presence of highly potent corticosteroid drugs prescribed. Of 193 drugs prescribed by generic names, 154 (79.8\%) were high potency corticosteroids. The prescriptions showed generic name in $193(52.9 \%)$ cases as compared to 172 (47.1\%) prescriptions were done with trade/ brand names. As compared to other studies where only $1.36 \%$ of the drugs were found in generic form, while rest was prescribed in brand names ${ }^{(12)}$, our study had $52.9 \%$ prescriptions by generic names.

Our study showed that of total 312 prescriptions included in the study, 365 corticosteroids were prescribed, with an average of 1.17 drugs/ patient. In disparity, a study done by W.M. Sweileh in Palestine, the total number of topical corticosteroid drugs prescribed for the 802 patients was 616. ${ }^{(14)}$ Prescriptions with generic drugs should be promoted. This will lead to uniformity to prescriptions; patients will get standard treatment at low cost which will lead to increase compliance from the side of patients and will help in inhibiting ill practices in health care.

Table no. 2 shows the routes of administration, with $76 \%$ of corticosteroids being prescribed topically, whereas $24 \%$ of all corticosteroids prescribed by oral/ parental and intralesional route. The topical corticosteroids were in prescribed in 2 vehicle forms creams (229) and ointments (27). Our study matches with the study done by Deepika Tikoo, et al. where topical route for corticosteroid was prescribed in $2340(87.7 \%)$ of the study population. ${ }^{(15)}$

Table no. 3 shows prescription pattern of corticosteroids by trade name. Out of 365 drugs prescribed, a total of $172(47.12 \%)$ were prescribed by trade name.

Topically Cutisoft $1 \%$ cream (21) and Relysal 6\% ointment (21) were most common drugs prescribed by their trade names, whereas orally Tab. Omnacortil (30) and parentally Inj. Kenacort (24) were the most commonly prescribed drugs by oral and parental routes. Major route of all the drugs prescribed with trade name was of topical application i.e., $106(61.6 \%)$, whereas other routes comprised of $66(38.4 \%)$ prescriptions. Majority of the drugs prescribed by trade names and in combinations (Fixed Dose Combinations), were in the category of Ultra/ Very high $88(51.16 \%)$ followed by medium potency $54(31.40 \%)$ and least by Low/ mild potency 30 (17.44\%). By combining both generic and trade named prescriptions, we conclude that $242(66.3 \%)$ were high potency, $81(22.2 \%)$ were medium and 42 (11.5\%) were low/ mild potency drugs. Our study findings supported by the observations on another study done by Bijoy KP et al. which showed that in about $43.47 \%$ instances high potency steroids were prescribed while steroids with mild potency were least prescribed (15.22\%). ${ }^{(16)}$

Table no. 3 shows that for total of 312 patients, 785 drugs were co-administered. Antihistaminic drugs were the most common co-administered drug i.e., 241 (30.7\%), followed by antibiotics 151 (19.23\%). Tab. Cetrizine 10mg 121 (50.2\%) and Tab. Chlorpheniramine maleate 4mg 66 (27.4\%) were the most common prescribed antihistaminics. In a study done $\mathrm{M}$ Ashok Kumar, in OPD antihistamines $(36.7 \%)$ followed by antibiotics $(15.4 \%)$ and emollients $(10.8 \%)$ were most commonly prescribed. ${ }^{(17)}$

Table no. 4 illustrates the contents of the prescription. In the patients details, name, age, 
sex, address and date of prescription was present in $100 \%$ of the study subjects. The occupation of the study subjects was seen on only $35.9 \%$ of prescriptions. The details pertaining to medication related information, dosage forms and dosage units were present on $100 \%$ of the prescriptions. Other details like the strength $(80.77 \%)$, frequency of administration (89.10\%), quantity (46, 55), Duration $(95.5 \%)$ were also of present. The details were aptly present on most of the prescriptions owing to computerised system of OPD and inpatient patient registration and drugs dispensing. Similarly, prescriber related information was also present in $100 \%$ of the prescriptions owing to the computerised system. Our study prescription contents are well compared to a study done by Purushotham et al. ${ }^{(18)}$

For successful treatment with TC, key factors to be considered are accurate diagnosis, selecting the correct drug, keeping in mind the potency, delivery vehicle, frequency of application, duration of treatment and adverse effects, and proper patient profiling. ${ }^{(19)}$

\section{Conclusion}

The prescription of corticosteroids is guided by coexisting diseases, so drugs which are prescribed should take care of coexisting diseases rather than aggravating those. This rational was found to be maintained in most of the prescriptions. In this study it was found that average number of drugs prescribed per prescription was 3.68, whilst the average corticosteroid prescribed was 1.17.

The results of the study were communicated to the dermatology department to promote rational use of drugs. The study is however not without limitations. The study was carried out only in dermatology OPD. Inpatients, casualties and emergency occurrences also needed to be studied for the same. Patient compliance of medications, knowledge, attitude and practices of the patient and prescriber, adverse drug reaction monitoring and cost analysis were not taken into account. More studies in other specialties and larger populations will further throw light on the prescription pattern trends and magnitude of irrationalities in the same.

With regard to inflammatory skin conditions and rise in prevalence of various etiological factors like the genetics, autoimmune factors, rising pollution, staggering population, poverty, increase demand and limited supply of services; regular audits, training and feedback should be taken place and should be followed. These will be important tools for checking irrational use of corticosteroids and other co-administered drugs.

Improving patients' knowledge, attitude and compliance towards the treatment can prove cornerstone in improving rational use of drugs. Prescribing by generic name should be encouraged. Prescribers should be reinvigorated in providing treatment following STGs (Standard Treatment Guidelines) and protocols.

\section{References}

1. Brazzini B, Pimpinelli N. New and established topical corticosteroids in dermatology. American journal of clinical dermatology. 2002 Feb 1;3(1):47-58.

2. Burkhart C, Morrell D, Goldsmith L. Dermatological Pharmacology. In: Brunton LL, Chabner BA, Knollmann BC, editors. Goodman \& Gilman's the Pharmacological basis of therapeutics. 12th ed. New Delhi: Mc Graw-Hill Publishers; 2011.1803-32.

3. Del Rosso J, Friedlander SF. Corticosteroids: options in the era of steroid-sparing therapy. Journal of the American academy of dermatology. 2005 Jul 31;53(1):S50-8.

4. Lee $\mathrm{M}$, Marks $\mathrm{R}$. The role of corticosteroids in dermatology. Australian Prescriber. 1998 Jan 1; 21(1): 9-11.

5. Ramam M, Kumrah L. Systemic Corticosteroid Therapy And The Hypothalamopituitary-Adrenal Axis. Indian Journal of Dermatology. 2001 Jan $1 ; 46(01): 1-7$. 
6. Shankar PR. Essential medicines and health products information portal. Journal of pharmacology \& pharmacotherapeutics. 2014 Jan;5(1):74-5.

7. Vandenbroucke JP, von Elm E, Altman DG, Gøtzsche PC, Mulrow CD, Pocock SJ, Poole C, Schlesselman JJ, Egger M. STROBE Initiative. Strengthening the Reporting of Observational Studies in Epidemiology (STROBE): explanation and elaboration. International journal of surgery. 2014 Dec 31;12(12):1500-24.

8. Israel GD. Sampling the evidence of extension program impact. University of Florida Cooperative Extension Service, Institute of Food and Agriculture Sciences, EDIS; 1992 Oct. (Last Accessed on 2018 September 12).

9. Israel GD. Determining sample size: University of Florida Cooperative Extension Service. Institute of Food and Agriculture Sciences, EDIS. 1992. (Last Accessed 2018 September 12).

10. Bylappa BK, Patil RT, Pillai RT. Drug prescribing pattern of topical corticosteroids in dermatology unit of a tertiary-care hospital. Int J Med Sci Public Health 2015;4:1702-1707.

11. Kasyap JS, Aiyappa C, Sumathy TK. Topical Corticosteroid Usage in Dermatology OPD in a Medical Teaching Hospital. Journal of Pharmaceutical Research. 2013 Sep 1;12(3):119-21.

12. Mukherjee S. Assessment of corticosteroid utilization pattern among dermatology outpatients in a tertiary care teaching hospital in Eastern India. International Journal of Green Pharmacy (IJGP). 2017 Jan 9;10(04).178-82.

13. Mahendra Kumar Jaiswal. Prescription audit of Corticosteroids in Dermatology OPD of a tertiary care teaching hospital of tribal region of central-south India.
International Journal of Biomedical Research. 2017; 8(01): 20-25.

14. Sweileh WM. Audit of prescribing practices of topical corticosteroids in outpatient dermatology clinics in north Palestine. Eastern Mediterranean Health Journal.2006;12(1/2):161-69.

15. Tikoo D, Chopra SC, Kaushal S, Dogra A. Evaluation of Drug Use Pattern in Dermatology as a Tool to Promote Rational Prescribing. JK Science. 2011 Sept;13(3):128-31.

16. Bijoy KP, Vidyadhar RS, Palak P, Chintan SP, Atmaram PP. Drug prescribing and economic analysis for skin diseases in dermatology OPD of an Indian tertiary care teaching hospital: a periodic audit. Indian journal of pharmacy practice. 2012;5(1):28-33.

17. Kumar AM, Noushad PP, Shailaja K, Jayasutha J, Ramasamy C. A study on drug prescribing pattern and use of corticosteroids in dermatological conditions at a tertiary care teaching hospital. Int J Pharm Sci Rev Res. 2011;9(2):132-5.

18. Purushotham K, Eesha B R. Prescription Trend of Topical Corticosteroids in Outpatient of Dermatology in a Tertiary Care Hospital in Tumakuru, Karnataka. Int J Pharmacol and Clin Sci. 2016;5(3):77-72.

19. Rathi SK, D’Souza P. Rational and ethical use of topical corticosteroids based on safety and efficacy. Indian journal of dermatology. 2012 Jul;57(4):251-59. 